Article

\title{
A Remark on Structure of Projective Klingenberg Spaces over a Certain Local Algebra
}

\author{
Marek JukliD \\ Department of Algebra and Geometry, Faculty of Science, Palacký University Olomouc, 17. Listopadu 12, \\ 71146 Olomouc, Czech Republic; marek.jukl@upol.cz
}

Received: 5 June 2019; Accepted: 30 July 2019; Published: 3 August 2019

\begin{abstract}
This article is devoted to the projective Klingenberg spaces over a local ring, which is a linear algebra generated by one nilpotent element. In this case, subspaces of such Klingenberg spaces are described. The notion of the "degree of neighborhood" is introduced. Using this, we present the geometric description of subsets of points of a projective Klingenberg space whose arithmetical representatives need not belong to a free submodule.
\end{abstract}

Keywords: projective Klingenberg space; point; hyperplane; local ring; plural algebra; $A$-space; $A$-subspace; neighbor points

\section{Introduction}

Projective Klingenberg spaces ( $P K S$ ) may be characterized as an incidence structure whose homomorphic image is a projective space over a field. Geometries over rings began to be studied systematically in the mid-2 $0^{\text {th }}$ Century. W. Klingenberg [1] followed the study of incidence structures of D. Barbilian [2] and introduced Klingenberg spaces (initially projective spaces with homomorphisms). The definition of a PKS of general finite dimension was presented by H. H. Lück in [3]. These topics were also developed by J. C. Ferrar and F. D. Veldkamp [4-6] and P. Y. Bacon (e.g., [7]). Projective geometry is also related to the theory of geodesic mappings (e.g., [8]). F. Machala [9] introduced projective Klingenberg spaces over local rings. The arithmetical fundament of such spaces is a free finite-dimensional $A$-module over a local ring $A$ ( $A$-space in the sense of B.R. McDonald [10]). Subspaces (points, lines, hyperplanes, etc.) of a PKS over a ring $A$ are subsets of points whose homomorphic images are subspaces of the respective dimension of the projective space over $A / \operatorname{Rad}(A)$ mentioned above. Thus, PKS may be also treated as a projective space over a ring in the sense of Bingen. In the case of PKS over certain local rings (plural algebras, [11]), we may study in more detail the structure of PKS, and we can find some special properties (for example, we introduce the degree of neighborhood). By this apparatus, we may describe not only subspaces of PKS, but also such sets of points (submodules of PKS) whose arithmetical representatives belong to a general submodule of the arithmetical fundament of PKS.

Let us consider the following local algebra $A$ (according to [11]):

Definition 1. A plural algebra of order $m$ over a field $T$ is every linear algebra $A$ on $T$ having as a vector space over T a basis:

$$
\left\{1, \eta, \eta^{2}, \ldots, \eta^{m-1}\right\} \text { with } \eta^{m}=0 .
$$

Remark 1. It follows from Definition 1 that any element $\alpha$ of $A$ may be uniquely expressed in the form:

$$
\alpha=\sum_{j=0}^{m-1} a_{j} \eta^{j} .
$$


Evidently, $A$ is a local ring with the maximal ideal $\mathfrak{a}=\eta A$, and all proper ideals of $A$ are just $\eta^{j} A, 1 \leq j \leq m$.

For the case $m=2, T=\mathbb{R}$, we obtain well-known dual numbers; for general $m$, we have plural numbers. Moreover, if $T=\mathbb{R}$, then $A$ is a Weil algebra of order $m$ and width one; therefore any $A$-space is also a Weil module.

Let $M$ be an $A$-space. It is well known that all bases of $M$ have the same number of elements (A-dimension of $M$ ), and from every set of generators, a basis of $M$ may be selected (see [10]). Above that, in our case, the following properties of $A$-space $M$ have been proven in [12] (the point 3 may be considered as a criterion of transversality of two $A$-subspaces; Veldkamp [13] called two $A$-subspaces transversal if both their intersection and their sum are $A$-subspaces):

Theorem 1. Let $M$ be an A-space over a plural algebra A. Then, the following hold:

1. Any linearly-independent system of elements of $M$ may be completed to a basis of $M$,

2. A submodule of $M$ is a free submodule if and only if it is a direct summand of $M$.

3. An intersection of two A-subspaces of $M$ is an A-subspace of $M$ if and only if the sum of them is an A-subspace of $M$.

Remark 2. A subspace of an A-space $M$ (A-subspace) is usually defined as a free direct summand of $M$. If $A$ is a plural algebra, it follows from Theorem 1 that $A$-subspaces of $M$ are just all free submodules of $M$.

Through the following text, let $M$ denote an arbitrary, but fixed $A$-space over a plural algebra $A$. Let us consider an endomorphism $\eta$ on $M$ defined by the relation:

$$
\forall \boldsymbol{x} \in M: \eta(\boldsymbol{x})=\eta \boldsymbol{x} .
$$

Theorem 2. Let $K$ be a nontrivial submodule of $A$-space $M$. Then, there exists a system $\mathcal{B}_{0}, \ldots, \mathcal{B}_{r}$ of subsets on $M$ such that:

1. $\mathcal{B}_{0} \cup \cdots \cup \mathcal{B}_{r-1} \cup \mathcal{B}_{r}$ is a basis of $M$,

2. $\eta^{m-r} \mathcal{B}_{0} \cup \eta^{m-r+1} \mathcal{B}_{1} \cup \cdots \cup \eta^{m-1} \mathcal{B}_{r-1}$ is a set of generators of $K$.

In this case, $r, 1 \leq r \leq m$, is an integer with $K \subseteq \operatorname{Ker} \eta^{r} \wedge K \not \subset \operatorname{Ker} \eta^{r-1}$.

Proof. Let us denote $\vartheta=\eta \mid K, \vartheta \in \operatorname{End}(K)$.

Since $M$ is a free module, it follows from [14] that $\eta^{j} M=\operatorname{Ker} \eta^{m-j}, 0 \leq j \leq m$. This implies:

$$
K \cap \eta^{j} M=\operatorname{Ker} \vartheta^{m-j}, 0 \leq j \leq m .
$$

Evidently, there exists a unique $r \in \mathbb{N}, 1 \leq r \leq m$, such that $K \subseteq \operatorname{Ker} \eta^{r} \wedge K \not \subset \operatorname{Ker} \eta^{r-1}$. Respecting the fact that $K$ is a vector space over $A / \mathfrak{a}=T$, we have that $\vartheta$ is a nilpotent endomorphism of $K$, and therefore, we obtain elements

$$
\begin{aligned}
& \boldsymbol{u}_{1}, \ldots, \boldsymbol{u}_{s_{0}}, \boldsymbol{u}_{s_{0}+1}, \ldots, \boldsymbol{u}_{s_{1}}, \boldsymbol{u}_{s_{1}+1}, \ldots, \boldsymbol{u}_{s_{2}}, \ldots, \boldsymbol{u}_{s_{r-2}+1}, \ldots, \boldsymbol{u}_{s_{r-1}} \text { of } K \text { such that: } \\
& \qquad \eta^{r-k} \boldsymbol{u}_{1}, \ldots, \eta^{r-k} \boldsymbol{u}_{s_{0}}, \eta^{r-k-1} \boldsymbol{u}_{s_{0}+1}, \ldots, \eta^{r-k-1} \boldsymbol{u}_{s_{1}}, \ldots, \boldsymbol{u}_{s_{r-k-1}+1}, \ldots, \boldsymbol{u}_{s_{r-k}}
\end{aligned}
$$

form a $T$-basis $\operatorname{Ker} \vartheta^{k} \bmod \operatorname{Ker} \vartheta^{k-1}, 1<k<r-1$.

As $\left\{\boldsymbol{u}_{1}, \ldots, \boldsymbol{u}_{s_{0}}\right\} \subseteq \operatorname{Ker} \vartheta^{r} \subseteq \eta^{m-r} M$ (see (3)), there exist $\boldsymbol{v}_{1}, \ldots, \boldsymbol{v}_{s_{0}} \in M$ with:

$$
\boldsymbol{u}_{i}=\eta^{m-r} \boldsymbol{v}_{i}, 1 \leq i \leq s_{0} .
$$

In the same way, $\left\{\boldsymbol{u}_{s_{r-k-1}+1}, \ldots, \boldsymbol{u}_{s_{r-k}}\right\} \subseteq \operatorname{Ker} \vartheta^{k}$ implies the existence of elements

$\boldsymbol{v}_{s_{r-k-1}+1}, \ldots, \boldsymbol{v}_{s_{r-k}} \in M$ with:

$$
\boldsymbol{u}_{i}=\eta^{m-k} \boldsymbol{v}_{i}, s_{r-k-1}+1 \leq i \leq s_{r-k}, \text { for } k=1, \ldots, r-1 .
$$


Let us denote $\mathcal{B}_{0}=\left\{\boldsymbol{v}_{1}, \ldots, \boldsymbol{v}_{s_{0}}\right\}$ and $\mathcal{B}_{r-k}=\left\{\boldsymbol{v}_{s_{r-k-1}+1}, \ldots, \boldsymbol{v}_{s_{r-k}}\right\}, k=r-1, \ldots, 1$.

(a) Let us show the linear independence of the set $\mathcal{B}_{0} \cup \ldots \cup \mathcal{B}_{r-1}$. Supposing:

$$
\sum_{i=1}^{s_{r-1}} \xi_{i} \boldsymbol{v}_{i}=\boldsymbol{o}
$$

and denoting (according to (1)) $\xi_{i}=\sum_{j=0}^{m-1} x_{i j} \eta^{j}, 1 \leq i \leq s_{r-1}$, where $x_{i j} \in T$, we obtain:

$$
\sum_{j=0}^{m-1} \eta^{j} \sum_{i=1}^{s_{r-1}} x_{i j} \boldsymbol{v}_{i}=\boldsymbol{o}
$$

Multiplying this equality by $\eta^{m-1}$ and using (4) and (5), we may write:

$$
\sum_{i=1}^{s_{0}} x_{i 0}\left(\eta^{r-1} \boldsymbol{u}_{i}\right)+\sum_{i=s_{0}+1}^{s_{1}} x_{i 0}\left(\eta^{r-2} \boldsymbol{u}_{i}\right)+\cdots+\sum_{i=s_{r-2}+1}^{s_{r-1}} x_{i 0} \boldsymbol{u}_{i}=\boldsymbol{o}
$$

We have obtained a linear combination of the elements of a $T$-basis of submodule $K$ with coefficients from $T$. It gives $x_{i 0}=0$, for $i=1, \ldots, s_{r-1}$. Therefore, (6) may be written in the form:

$$
\sum_{j=1}^{m-1} \eta^{j} \sum_{i=1}^{s_{r-1}} x_{i j} \boldsymbol{v}_{i}=\boldsymbol{o}
$$

Multiplying this equality by $\eta^{m-2}$ and using again (4) and (5), we obtain $x_{i 1}=0, i=1, \ldots, s_{r-1}$. Then, (6) may be written as:

$$
\sum_{j=2}^{m-1} \eta^{j} \sum_{i=1}^{s_{r-1}} x_{i j} \boldsymbol{v}_{i}=\boldsymbol{o}
$$

Multiplying (6) by $\eta^{m-3}, \ldots, \eta$, consecutively, we have that all $x_{i j}$ are zero and $\xi_{1}=\xi_{2}=\cdots=\xi_{s_{r-1}}=$ 0 , consequently.

Since $\mathcal{B}_{0} \cup \ldots \cup \mathcal{B}_{r-1}$ is a set of linearly-independent elements, it may be completed by a set $\mathcal{B}_{r}$ to an $A$-basis of $M$ (according to Theorem 1).

(b) Let us show that $\eta^{m-r} \mathcal{B}_{0} \cup \eta^{m-r+1} \mathcal{B}_{1} \cup \cdots \cup \eta^{m-1} \mathcal{B}_{r-1}$ generates (over $A$ ) the module $K$. Respecting (4) and (5), we may write:

$$
\begin{aligned}
& \boldsymbol{x}=\sum_{\substack{1 \leq i \leq s_{0} \\
0 \leq j \leq r-1}} \mathbf{x}_{i j}\left(\eta^{j} \boldsymbol{u}_{i}\right)+\sum_{\substack{s_{0}<i \leq s_{1} \\
0 \leq j \leq r-2}} x_{i j}\left(\eta^{j} \boldsymbol{u}_{i}\right)+\cdots+\sum_{\substack{s_{r-3}<i \leq s_{r-2} \\
0 \leq j \leq 1}} x_{i j}\left(\eta^{j} \boldsymbol{u}_{i}\right)+ \\
& +\sum_{s_{r-2}<i \leq s_{r-1}} x_{i 0} \boldsymbol{u}_{i}= \\
& =\sum_{\substack{1 \leq i \leq s_{0} \\
0 \leq j \leq r-1}} x_{i j}\left(\eta^{j+m-r} \boldsymbol{v}_{i}\right)+\sum_{\substack{s_{0}<i \leq s_{1} \\
0 \leq j \leq r-2}} x_{i j}\left(\eta^{j+m-r+1} \boldsymbol{v}_{i}\right)+\cdots+ \\
& +\sum_{\substack{s_{r-3}<i \leq s_{r-2} \\
0 \leq j \leq 1}} x_{i j}\left(\eta^{j+m-2} \boldsymbol{v}_{i}\right)+\sum_{s_{r-2}<i \leq s_{r-1}} x_{i 0} \eta^{m-1} \boldsymbol{v}_{i}= \\
& =\sum_{\substack{1 \leq i \leq s_{0} \\
0 \leq k \leq m-1}}\left(x_{i k} \eta^{k}\right)\left(\eta^{m-r} \boldsymbol{v}_{i}\right)+\sum_{\substack{s_{0}<i \leq s_{1} \\
0 \leq k \leq m-1}}\left(x_{i k} \eta^{k}\right)\left(\eta^{m-r+1} \boldsymbol{v}_{i}\right)+\cdots+ \\
& +\sum_{\substack{s_{r-3}<i \leq s_{r-2} \\
0 \leq k \leq m-1}}\left(x_{i k} \eta^{k}\right)\left(\eta^{m-2} \boldsymbol{v}_{i}\right)+\sum_{\substack{s_{r-2}<i \leq s_{r-1} \\
0 \leq k \leq m-1}}\left(x_{i k} \eta^{k}\right)\left(\eta^{m-1} \boldsymbol{v}_{i}\right)=
\end{aligned}
$$




$$
\begin{gathered}
=\sum_{1 \leq i \leq s_{0}} \xi_{i}\left(\eta^{m-r} \boldsymbol{v}_{i}\right)+\sum_{s_{0}<i \leq s_{1}} \xi_{i}\left(\eta^{m-r+1} \boldsymbol{v}_{i}\right)+\cdots+ \\
+\sum_{s_{r-3}<i \leq s_{r-2}} \xi_{i}\left(\eta^{m-2} \boldsymbol{v}_{i}\right)+\sum_{s_{r-2}<i \leq s_{r-1}} \xi_{i}\left(\eta^{m-1} \boldsymbol{v}_{i}\right) .
\end{gathered}
$$

If an element $\boldsymbol{x}$ of $M$ may be expressed as a linear combination of elements of a $T$-basis of submodule $K$ with coefficients from $T$, then it may be also expressed as a linear combination of the elements of the set $\eta^{m-r} \mathcal{B}_{0} \cup \eta^{m-r+1} \mathcal{B}_{1} \cup \cdots \cup \eta^{m-1} \mathcal{B}_{r-1}$ with coefficients from $A$ and vice versa.

\section{Projective Klingenberg Spaces}

Let us remind about the definition of a projective Klingenberg space (Klingenberg's definition as modified by Lück [3]).

Definition 2. A projective Klingenberg space of dimension $n, n \geq 2$, is an incidence structure $\mathcal{P}=(P, H, I)$ with a homomorphism $\mu$ of $\mathcal{P}$ onto an n-dimensional projective space $\mathcal{P}_{0}=\left(P_{0}, H_{0}, I_{0}\right)$ such that:

1. If $X_{1}, \ldots, X_{k}, 1 \leq k \leq n$, are points in $P$ such that $\mu\left(X_{1}\right), \ldots, \mu\left(X_{k}\right)$ are independent in $P_{0}$, then there exists a hyperplane $\mathcal{H}$ in $H$ such that $X_{1}, \ldots, X_{k} I \mathcal{H}$. This hyperplane is unique if $k=n$.

2. this condition is the dual of one.

3. If $X_{1}, \ldots, X_{n-1} \in P$ and $\mathcal{H}_{1}, \mathcal{H}_{2} \in H$ are such that $\mu\left(X_{1}\right), \ldots, \mu\left(X_{n-1}\right) \in P_{0}$, as well as $\mu\left(H_{1}\right), \mu\left(H_{2}\right) \in H_{0}$ are independent and $P_{1}, \ldots, P_{n-1} I \mathcal{H}_{1}, \mathcal{H}_{2}$, then: $\left(Y I \mathcal{H}_{1}, \mathcal{H}_{2} \wedge P_{1}, \ldots, P_{n-1} I \mathcal{H}\right) \Rightarrow Y I \mathcal{H}$.

Following [9], we define:

Definition 3. Let $A$ be a local ring and $\mathfrak{a}$ be its maximal ideal. Let us denote $M=A^{n+1}, n \geq 2, \bar{M}=M / \mathfrak{a} M$. Then, an incidence structure $\mathcal{P}_{A}$ such that

1. points in $\mathcal{P}_{A}$ are just all submodules $[\boldsymbol{x}] \subseteq M$ such that $\langle\boldsymbol{x}\rangle$ is a nonzero element of $\bar{M}$,

2. hyperplanes in $\mathcal{P}_{A}$ are just all submodules $\left[\boldsymbol{u}_{1}, \boldsymbol{u}_{2} \ldots \boldsymbol{u}_{n}\right] \subseteq M$ such that $\left\langle\boldsymbol{u}_{1}\right\rangle,\left\langle\boldsymbol{u}_{2}\right\rangle, \ldots,\left\langle\boldsymbol{u}_{n}\right\rangle$ are linearly-independent elements of $\bar{M}$,

3. the incidence relation is an inclusion,

is called an n-dimensional projective Klingenberg space over the ring A.

For any point $X=[\boldsymbol{x}]$ of $\mathcal{P}_{A}$, an element $\boldsymbol{x}$ is called an arithmetical representative of the point $X$. The module $M$ is called the arithmetical fundament of the space $\mathcal{P}_{A}$.

It is known that for $n \geq 3$, there is no (up to an isomorphism) other projective Klingenberg spaces except of PKS over a local ring. In the case of planes, it is true only for Desarguesian ones.

Remark 3. Let us consider the n-dimensional projective space $\mathcal{P}_{(A / \mathfrak{a})}$ over the field $A / \mathfrak{a}$ (i.e., with arithmetical fundament $\bar{M}=M / \mathfrak{a} M)$. Then, $\mathcal{P}_{(A / \mathfrak{a})}$ is an homomorphic image of $\mathcal{P}_{A}$ in the homomorphism $\mu$ defined by:

$$
\forall X, X=[\boldsymbol{x}], \boldsymbol{x} \in M: \mu(X)=[\langle\boldsymbol{x}\rangle] .
$$

Thus, points of $\mathcal{P}_{A}$ are just submodules in $M$ that are (by homomorphism $\mu$ ) mapped on points of the projective space $\mathcal{P}_{(A / \mathfrak{a})}$, and hyperplanes in $\mathcal{P}_{A}$ are just submodules in $M$ that are mapped (by homomorphism $\mu$ ) on hyperplanes of the projective space $\mathcal{P}_{(A / \mathfrak{a})}$.

The following definition is natural. According to Definition 3, points and hyperplanes correspond to the cases $k=0$ and $k=n-1$, respectively (it is usual to identify a hyperplane $\mathcal{H}$ with the set of points incident with $\mathcal{H}$, and we will use this approach). 
Definition 4. Let $\mathcal{P}_{A}$ be an $n$-dimensional projective Klingenberg space and $M$ be its arithmetical fundament. Let a submodule $K$ of $M$ be given. A set:

$$
\mathcal{K}=\left\{X \in \mathcal{P}_{A}, X=[\boldsymbol{x}]: \boldsymbol{x} \in K\right\}
$$

is called a $k$-dimensional subspace in $\mathcal{P}_{A}, 0 \leq k \leq n-1$, if $K=\left[\boldsymbol{u}_{1}, \boldsymbol{u}_{2} \ldots \boldsymbol{u}_{k+1}\right]$, and $\left\langle\boldsymbol{u}_{1}\right\rangle,\left\langle\boldsymbol{u}_{2}\right\rangle, \ldots,\left\langle\boldsymbol{u}_{k+1}\right\rangle$ are linearly-independent elements of $\bar{M}$.

The submodule $K$ is called an arithmetical fundament of the subspace $\mathcal{K}$.

Define a relation "to be neighbor" on points of PKS. Thus, PKS may be also treated as Barbilian spaces.

Definition 5. Points $X, Y \in \mathcal{P}_{A}$ are called neighbors, if $\mu(X)=\mu(Y)$. Otherwise, we speak of non-neighbor points.

In what follows, we study $n$-dimensional $\mathrm{PKS} \mathcal{P}_{A}$ over a plural algebra $A$ with arithmetical fundament denoted by $M$ (Definition 3). Obviously, the module $M$ is an $A$-space. Using Theorem 1 (and Theorem I.2 of [10]), we have characterized the notions point and hyperplane, as well as the subspace of arbitrary dimension in PKS (cf. [15]).

Theorem 3. Let $\mathcal{P}_{A}$ be a projective Klingenberg space. Then, $k$-dimensional subspaces of $\mathcal{P}_{A}, 0 \leq k \leq n-1$, are just all subsets (8) such that $K$ is a $(k+1)$-dimensional $A$-subspace in $M$.

Theorem 3 implies that any $\mathrm{PKS}_{\mathcal{P}_{A}}$ can be also treated as a projective space over the ring $A$ in the sense of Bingen [16] if we ignore the neighbor relation. Bingen has defined these projective spaces as structures formed by $A$-subspaces of a free finite-dimensional $A$-module.

Let us define a useful subrelation of the relation "to be neighbor". We get a new equivalence relation on the set of points of $\mathcal{P}_{A}$; a couple of points belongs to this one, if they have the same degree of neighborhood. That allows one to study new properties of PKS.

Definition 6. [15] Let $X=[\boldsymbol{x}]$ and $Y=[\boldsymbol{y}]$ be points of a projective Klingenberg space $\mathcal{P}_{A}$, and let $r$ be a non-negative integer satisfying:

$$
\left(\eta^{m-r} \boldsymbol{x} \in[\boldsymbol{y}]\right) \wedge\left(\eta^{m-r-1} \boldsymbol{x} \notin[\boldsymbol{y}] \vee r=m\right) .
$$

The number $r$ is called the degree of neighborhood of the points $X$ and $Y$.

Clearly, for a couple of non-neighbor points, we have $r=0$, for neighbor different points $1 \leq r \leq m-1$ and for identical points $r=m$.

For arbitrary point $X$ and every integer $r, 0 \leq r \leq m$, there exists at least one point $Y$ such that the degree of neighborhood of points $X, Y$ is equal to $r$.

Definition 7. Let $X$ be a point and $\mathcal{K}$ be a set of points of a projective Klingenberg space. We said that $r$ is a degree of neighborhood of $X$ and $\mathcal{K}$ if there exists at least one point $Y \in \mathcal{K}$ such that the degree of the neighborhood of points $X, Y$ is equal to $r$ and the degree of the neighborhood of $X$ and any point of $\mathcal{K}$ is no greater then $r$.

Corollary 1. If $\mathcal{K}$ is a subspace of $\mathcal{P}_{A}$ and $K$ is an arithmetical fundament of $\mathcal{K}$, then the degree of neighborhood of a point $X=[\boldsymbol{x}]$ and subspace $\mathcal{K}$ is equal to $r$ if and only if $\left(\left(\eta^{m-r} \boldsymbol{x} \in K\right) \wedge\left(\eta^{m-r-1} \boldsymbol{x} \notin K \vee r=m\right)\right)$.

Let $\phi$ be a linear form on an $h$-dimensional $A$-space $M$ such that $\operatorname{Im} \phi \not \subset \mathfrak{a}$. Then, $\operatorname{Ker} \phi$ is an $(h-1)$-dimensional $A$-subspace in $M$ (see [12]), and for $K=\operatorname{Ker} \phi$, the subset (8) forms a hyperplane 
in $\operatorname{KPS} \mathcal{P}_{A}$. If $\phi$ is a general linear form, then its kernel need not be an $A$-subspace. Then, there is a question about how to describe a set of points of $\mathcal{P}_{A}$ whose arithmetical representatives belong to a kernel of a general linear form.

For any linear form $\phi$ on $M$, there exists a linear form $\phi_{0}$ with $\operatorname{Im} \phi_{0} \not \subset \mathfrak{a}$ and just one integer $k, 0 \leq k \leq m$, (called an order of linear form $\phi$ ) such that $\phi=\eta^{k} \phi_{0}$ (see [17]).

Lemma 1. Let $\mathcal{P}_{A}$ be projective Klingenberg space. Let $\phi$ be an arbitrary linear form on $M$ of order $k$. Then, the set:

$$
\mathcal{H}=\left\{X \in \mathcal{P}_{A}, X=[\boldsymbol{x}]: \boldsymbol{x} \in \operatorname{Ker} \phi\right\}
$$

is formed by all points with the degree of neighborhood at least $m-k$ to a certain hyperplane $\mathcal{H}_{0}$ of $\mathcal{P}_{A}$. If $\phi_{0}$ is a form of zero order such that $\phi=\eta^{k} \phi_{0}$, then:

$$
\mathcal{H}_{0}=\left\{X \in \mathcal{P}_{A}, X=[\boldsymbol{x}]: \boldsymbol{x} \in \operatorname{Ker} \phi_{0}\right\} .
$$

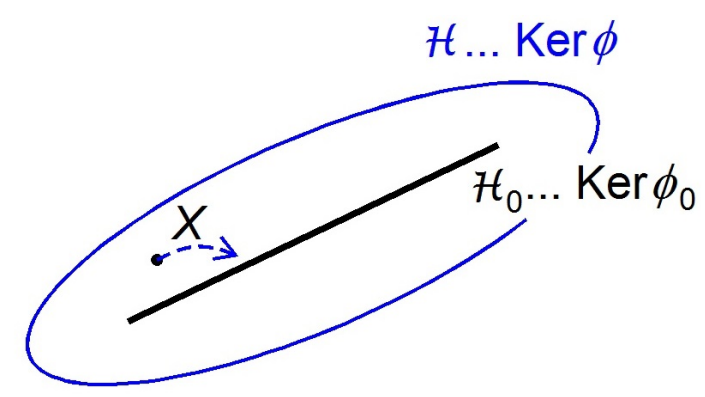

Proof. Let $\phi=\eta^{k} \phi_{0}$, where $\phi_{0}$ is a linear form of zero order. For kernels of linear forms $\phi, \phi_{0}$, it holds:

$$
\operatorname{Ker} \phi=\left\{\boldsymbol{x} \in M: \eta^{k} \boldsymbol{x} \in \operatorname{Ker} \phi_{0}\right\} .
$$

According to Theorem 3, a subset $\mathcal{H}_{0}$ is a hyperplane in $\mathcal{P}_{A}$. Let $X=[\boldsymbol{x}]$ be a point belonging to $\mathcal{H}$; thus, $\eta^{k} \boldsymbol{x} \in \operatorname{Ker} \phi_{0}$. Therefore, there exists an element $\boldsymbol{y} \in \operatorname{Ker} \phi_{0}$ with $\eta^{k} \boldsymbol{x}=\boldsymbol{y}$. Because $\operatorname{Ker} \phi_{0}$ is an $A$-subspace, $\boldsymbol{y}$ may be written as $\boldsymbol{y}=\eta^{l} \boldsymbol{z}$, where $\boldsymbol{z}$ is a linearly-independent element from Ker $\phi_{0}$ (clearly, $l=k$ ). We get $\eta^{k} \boldsymbol{x} \in[\boldsymbol{z}]$, which means that points $X$ and $Z, Z=[\boldsymbol{z}], Z \in \mathcal{H}_{0}$ are neighbors with degree at least $m-k$.

Now, we may present a natural generalization of the notion subspace of Klingenberg projective space $\mathcal{P}_{A}$. We have seen that subspaces in $\mathcal{P}_{A}$ are just all subsets of points whose arithmetical representatives belong to some $A$-subspace of $M$. Let us in (8) consider an arbitrary submodule $K$ of $M$. In this case, the subset (8) of points of $\mathcal{P}_{A}$ is appropriate, called a submodule of KPS.

Definition 8. Let $\mathcal{P}_{A}$ be an n-dimensional projective Klingenberg space and $M$ be its arithmetical fundament. Let a submodule $K$ of $M$ be given. Then, a set:

$$
\mathcal{K}=\left\{X \in \mathcal{P}_{A}, X=[\boldsymbol{x}]: \boldsymbol{x} \in K\right\}
$$

is called a submodule in $\mathcal{P}_{A}$ (with arithmetical fundament $K$ ).

The following theorem brings a pure geometric description of the submodules of PKS $\mathcal{P}_{A}$.

Theorem 4. Let $\mathcal{P}_{A}$ be projective Klingenberg space and $M$ be its arithmetical fundament. Let $K$ be an arbitrary non-trivial submodule in $M$. Then, there exists a system 


$$
\boldsymbol{H}_{0}, \ldots, \boldsymbol{H}_{r-1}, \boldsymbol{H}_{r}
$$

of sets of hyperplanes of $\mathcal{P}_{A}$ such that the submodule of $\mathcal{P}_{A}$ with arithmetical fundament $K$ is equal to the intersection of:

(0) all hyperplanes belonging to $\boldsymbol{H}_{r}$,

(1) a set of points having the degree of neighborhood at least $m-1$ to every hyperplane belonging to $\boldsymbol{H}_{r-1}$,

(r) a set of points having the degree of neighborhood at least mr to every hyperplane belonging to $\boldsymbol{H}_{0}$.

Proof. Let us denote by $\mathcal{K}$ the submodule of $\mathcal{P}_{A}$ with arithmetical fundament $K$. Due to Theorem 2, we get for a submodule $K$ a system of sets $\mathcal{B}_{0}, \ldots, \mathcal{B}_{r}$. Let us denote their elements in the following way:

$$
\mathcal{B}_{0}=\left\{\boldsymbol{e}_{i}\right\}_{i=1}^{k_{0}}, \mathcal{B}_{1}=\left\{\boldsymbol{e}_{i}\right\}_{i=k_{0}+1}^{k_{1}}, \ldots, \mathcal{B}_{r}=\left\{\boldsymbol{e}_{i}\right\}_{i=k_{r-1}}^{k_{r}}
$$

and construct by these sets a base $\mathcal{B}$ of an arithmetical fundament $M$. A dual base $\tilde{\mathcal{B}}$ of a dual $A$-space $\tilde{M}$ may be constructed as well (cf. [12]). Any element of $\tilde{\mathcal{B}}$ is a linear form of zero order, of course.

For any linear form $\tilde{\boldsymbol{e}}_{i}, 1 \leq i \leq n$, let us denote by $\mathcal{H}_{i}$ a hyperplane in $\mathcal{P}_{A}$ with arithmetical fundament $\operatorname{Ker} \tilde{e}_{i}$. We obtain the following system of sets of hyperplanes:

$$
\boldsymbol{H}_{0}=\left\{\mathcal{H}_{i}\right\}_{i=1}^{k_{0}}, \boldsymbol{H}_{1}=\left\{\mathcal{H}_{i}\right\}_{i=k_{0}+1}^{k_{1}}, \ldots, \boldsymbol{H}_{r}=\left\{\mathcal{H}_{i}\right\}_{i=k_{r-1}}^{k_{r}} .
$$

Let us consider an arbitrary point $X \in \mathcal{P}_{A}, X=[\boldsymbol{x}]$. Respecting the form of a set of generators of the submodule $K$, as is described in Theorem 2 (2), we have that $X$ belongs to $\mathcal{K}$ (i.e., $\boldsymbol{x} \in K$ ) if and only if:

$$
\begin{gathered}
\left\{\tilde{\boldsymbol{e}}_{i}(\boldsymbol{x}) \in \mathfrak{a}^{m-r}, 1 \leq i \leq k_{0}\right\} \wedge\left\{\tilde{\boldsymbol{e}}_{i}(\boldsymbol{x}) \in \mathfrak{a}^{m-r+1}, k_{0}+1 \leq i \leq k_{1}\right\} \wedge \cdots \wedge \\
\wedge\left\{\tilde{\boldsymbol{e}}_{i}(\boldsymbol{x}) \in \mathfrak{a}^{m-1}, k_{r-2}+1 \leq i \leq k_{r-1}\right\} \wedge\left\{\tilde{\boldsymbol{e}}_{i}(\boldsymbol{x})=0, k_{r-1}+1 \leq i \leq k_{r}\right\} .
\end{gathered}
$$

It may be equivalently expressed by:

$$
\begin{aligned}
& \left\{\eta^{r} \tilde{\boldsymbol{e}}_{i}(\boldsymbol{x})=0,1 \leq i \leq k_{0}\right\} \wedge\left\{\eta^{r-1} \tilde{\boldsymbol{e}}_{i}(\boldsymbol{x})=0, k_{0}+1 \leq i \leq k_{1}\right\} \wedge \cdots \wedge \\
& \wedge\left\{\eta \tilde{\boldsymbol{e}}_{i}(\boldsymbol{x})=0, k_{r-2}+1 \leq i \leq k_{r-1}\right\} \wedge\left\{\tilde{\boldsymbol{e}}_{i}(\boldsymbol{x})=0, k_{r-1}+1 \leq i \leq k_{r}\right\} .
\end{aligned}
$$

Finally, we have that $X \in \mathcal{K}$ if and only if:

$$
\begin{aligned}
& \left\{\boldsymbol{x} \in \operatorname{Ker} \eta^{r} \tilde{\boldsymbol{e}}_{i}, 1 \leq i \leq k_{0}\right\} \wedge\left\{\boldsymbol{x} \in \operatorname{Ker} \eta^{r-1} \tilde{\boldsymbol{e}}_{i}, k_{0}+1 \leq i \leq k_{1}\right\} \wedge \cdots \wedge \\
& \wedge\left\{\boldsymbol{x} \in \operatorname{Ker} \eta \tilde{\boldsymbol{e}}_{i}, k_{r-2}+1 \leq i \leq k_{r-1}\right\} \wedge\left\{\boldsymbol{x} \in \operatorname{Ker} \tilde{\boldsymbol{e}}_{i}, k_{r-1}+1 \leq i \leq k_{r}\right\} .
\end{aligned}
$$

Now, using Lemma 1, we obtain the proven theorem.

The notion of the "degree of neighborhood" has shown up as a key one, which allows us to describe pure geometrically point sets that form submodules of PKS. Now, we see that the structure of Klingenberg spaces $\mathcal{P}_{A}$ is richer than the structure of subspaces of a projective space over the ring $A$ in the sense of Bingen.

Funding: This research received no external funding.

Acknowledgments: This article is supported by IGA PrF 2019015 Palacký University, Olomouc, Czech Republic.

Conflicts of Interest: The author declares no conflict of interest. 


\section{References}

1. Klingenberg, W. Projektive Geometrien mit Homomorphismus. Math. Ann. 1956, 132, 180-200. [CrossRef]

2. Barbilian, D. Zur Axiomatik der projektiven ebenen Ringgeometrien, I. Jahresber. Deutsch. Math.-Ver. 1940, 50, 179-229.

3. Lück, H.H. Projektive Hjelmslevräume. J. Reine Angew. Math. 1970, 243, 121-158.

4. Ferrar, J.C.; Veldkamp, F.D. Neighbor-preserving homomorphisms between ring planes. Geom. Dedic. 1985, 18, 11-33. [CrossRef]

5. Veldkamp, F.D. Projective planes over rings of stable rank 2. Geom. Dedic. 1981, 11, 285-308. [CrossRef]

6. Veldkamp, F.D. Projective Barbilian spaces. Result. Math. 1987, 12, 434-449. [CrossRef]

7. Bacon, P.Y. An Introduction to Klingenberg Planes. Vol. I.-III; Bacon: Gainesville, FL, USA, 1979.

8. Mikeš, J.; Stepanova, E.; Vanžurová, A.; Sándor, B.; Berezovski, V.; Chepurna, E.; Chodorová, M.; Chudá, H.; Gavrilchenko, M.; Haddad, M.; et al. Differential Geometry of Special Mappings; Palacký University: Olomouc, Czech Republic, 2015.

9. Machala, F. Fundamentalsätze der projektiven Geometrie mit Homomorphismus. Rozpravy ČSAV Řada Mat. Př́rod. Věd 1980, 90, 5.

10. McDonald, B.R. Geometric Algebra over Local Rings; M. Dekker: New York, NY, USA, 1976.

11. Burgetová, R.; Klucký, D. The spectrum of a Cartesian product of plural algebras. Časopis Pěstování Matematiky 1981, 106, 402-406.

12. Jukl, M. Grassman formula for certain type of modules. Acta Univ. Palacki. Olomuc. Math. 1995, 34, 69-74.

13. Veldkamp, F.D. Geometry over rings. In Handbook of Incidence Geometry; Elsevier: Amsterdam, The Netherlands; Lausanne, Switzerland; New York, NY, USA, 1994; pp. 1033-1085.

14. Klucký, D. A contribution to the theory of modules over finite dimensional linear algebras. Časopis Estování Matematiky 1984, 109, 113-117.

15. Jukl, M. On homologies of Klingenberg projective spaces over special commutative local rings. Publ. Math. Univ. Debreciensis 1999, 55, 113-121.

16. Bingen, F. Geometrie projective sur un anneau semiprimaire. Acad. R. Belg. Bull. Cl Sci. 1966, 52, 13-24.

17. Jukl, M. Linear forms on free modules over certain local ring. Acta Univ. Palacki Olomuc. Math. 1993, 32, $49-62$.

(C) 2019 by the authors. Licensee MDPI, Basel, Switzerland. This article is an open access article distributed under the terms and conditions of the Creative Commons Attribution (CC BY) license (http:/ / creativecommons.org/licenses/by/4.0/). 